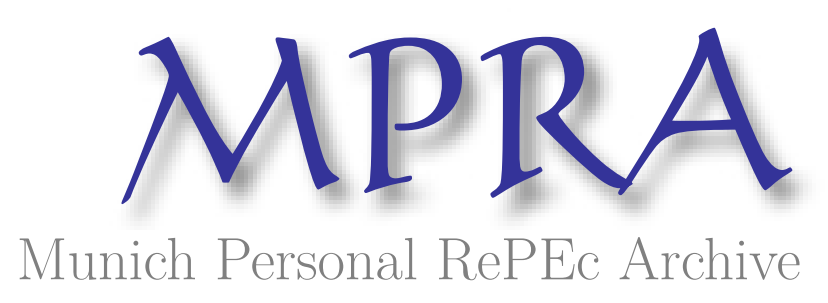

\title{
Constructing a GDP-based Index for Use as Benchmark
}

Cohen, Ruben D

October 2009

Online at https://mpra.ub.uni-muenchen.de/18390/

MPRA Paper No. 18390, posted 10 Nov 2009 00:18 UTC 


\title{
Constructing a GDP-based Index for Use as Benchmark
}

\author{
Ruben D. Cohen ${ }^{1,2}$
}

\begin{abstract}
The gross domestic product $[G D P]$ is a fundamental economic indicator that is frequently used as a benchmark for local equity indices. The widespread appeal of this association is understandable because an equity index, especially if broad, could, like the GDP, also manifest the state of the economy. At the same time, however, the validity of a direct relation between the two is debatable since the GDP is known to be characteristically different from the typical equity index, however broad.

In this work, we review some of the key elements that separate the GDP from a typical broad equity index in order to explain why the two cannot be compared directly with each other. We then incorporate a readily available mapping technique to create a $G D P$-based index that circumvents their inherent disparities and, thus, enable us to benchmark one against the other.
\end{abstract}

\section{Introduction}

The GDP, in level or growth rate, is one of the most commonly used indicators that reflect the state of a country's economy. There is no need to describe here how this parameter is defined or estimated, as there is an extensive literature that covers it. What is relevant to this work is that this measure, which is, in one form or another, used as a benchmark for various local equity indices, begins first as a forecast over some time horizon, which is soon followed by a revision. The closed-loop revising process continues iteratively until the target date is reached, by which time convergence occurs. At this stage, the GDP becomes realized and, thus, "historical" and the process repeats itself with a new prediction towards a new target date. The time span for this cycle, from prediction to convergence, normally equals one quarter, which is the reporting time scale for many economic indicators.

The drive to speculate on what the future holds for the $G D P^{3}$, as well as many other economic indicators, is fuelled by a society's need to invest and grow A correct guess could guide one to the right investment decision, which would, in turn, lead to

\footnotetext{
${ }^{1}$ Citi, London E14 5LB, UK.

${ }^{2}$ I express these views as an individual, not as a representative of companies with which I am connected. ${ }^{3}$ In fact, there are publications that specialize in consensus forecasting, with the objective of narrowing down the views of economists and analysts to bring them into focus.
} 
higher productivity, growth in real profits and, subsequently, personal wealth. This speculative approach to linking the $G D P$ with profits remains, in fact, essential to the development of any competitive economy that thrives on investment and growth. At the other extreme, also, after the speculations have been revised over and over and the true GDP number comes to light, it will, purely by construct, embody the profits that underlie its constituents.

The circular relationship that the $G D P$ has with profits, as summarised above, is, therefore, one reason why the indicator is used regularly as a benchmark for "broad“4 equity indices [Faugère \& Van Erlach (2006), Honnerová (2003), and many others]. Examples of such comparisons abound, whereby one simply has to open a financial publication [newspaper, magazine, etc.] to find them.

Considering all this, however, there is still the issue that a direct relationship between the two is not universally recognized (Kerschner et al, 1999). There are several reasons for this, among which are that (1) the GDP is based mainly on revenues, whereas equity valuation, the very basis of any equity index, centers on profits, (2) a typical equity index has a higher exposure to foreign earnings than does the $G D P,(3)$ the $G D P$ level, or index, is measured in units of income [i.e. \$/year], whereas an equity index is denominated in value [i.e. \$] and last, but not least, (4) by the time the GDP converges from speculation to historical, which could take up to several months depending on the forecast horizon, the number becomes actual. This contrasts sharply to what an equity index symbolizes, which is merely a speculation on the underlying firms' earnings potential going forward.

On a macro level, one could, more or less, get around statements (1) and (2) above, but not (3) and (4). In reference to (1), for instance, one could claim that a company's expenses comprise another company's profits, so that a merger between the two can create a conglomeration that generates only revenues. Thus, while taking the two companies separately is akin to an equity index whose overall value is determinable by individual profits, their merger becomes analogous to the $G D P$, which tracks revenues.

\footnotetext{
${ }^{4}$ By broad, we mean an equity index that comprises a large number of public companies, belonging to a broad range of sectors and specializing in a broad range of products. Thus, the broader the index, the closer it comes to representing the economy as a whole.
} 
Similarly, one could argue against (2) in that just as companies compete against each other economically, jurisdictions do so as well, but on a grander scale. For example, while an equity index in Country $A$, with an exposure to Country $B$, would contribute to, or gain from, the $G D P$ of Country $B$, an equity index in Country $B$, with an exposure to Country $A$, would contribute to, or gain from, Country $A$ 's GDP. This closed cycle, therefore, would have a compensating effect on an equity index, enabling its foreign exposure to circle back into the GDP and, subsequently, the local equity indices and vice versa. As result, one should, effectively, be able to compare, albeit not directly, the characteristics of a country's GDP to the local, broad indices.

Statements (3) and (4), on the other hand, hinge on deeper fundamental discrepancies between the GDP level and an equity index and, thus, are more challenging to crack. Here, as a consequence, one must follow a different path when trying to correlate the two parameters. Establishing this path and providing evidence for it, with intent to develop a $G D P$-based index for benchmarking purposes, constitute the remainder of this paper.

\section{Relating the GDP With an Equity Index}

There are, as mentioned above, two primary reasons ${ }^{5}$ why the GDP cannot serve directly as a benchmark for an equity index. Firstly, different units of measure characterize the two parameters and, secondly, while the GDP reflects an actual, quantifiable number, the value of an equity index is entirely speculative. Proof for the latter can be observed in Figure 1, where the historical level of the $U S$ nominal ${ }^{6} G D P$ is displayed alongside the $S \& P 500^{7}$ index, both plotted from 1975 to 2008 . Here, although there is similarity in long-term trends, one could easily spot the difference in volatility between the two quantities, where the higher volatility in the $S \& P 500$ is a manifestation of the speculations that shape it. In consequence, one must rely on an alternative way to accomplish the task of benchmarking an equity index, a speculative-based measure,

\footnotetext{
${ }^{5}$ As given by statements (3) and (4) in the previous section.

${ }^{6}$ Nominal instead of real because the equity index is in nominal terms.

${ }^{7}$ Although the DJIA index is highly correlated with the $S \& P 500$ and could have been used here to illustrate our example equally well, we have chosen to work with the latter primarily because it is broader and also used more popularly as a benchmark in a large number of studies.
} 
against the $G D P$, which is a realized number, not to mention the other differences already listed above.

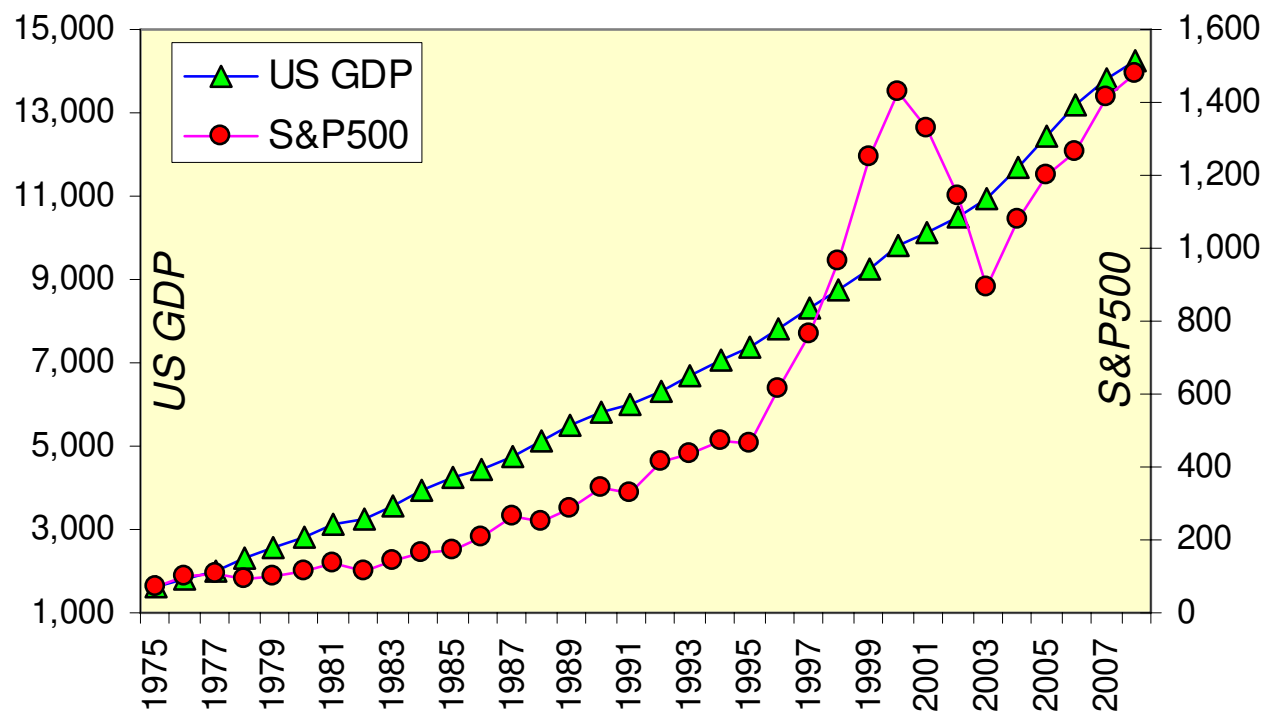

Figure 1 - Comparing the trends of the nominal US GDP level and S\&P500 index between 1975 and 2008. The higher volatility in the latter is a manifestation of its underlying speculative nature.

The alternative being considered here is founded on a readily existing methodology that can be also used to assess the relative valuation between the nominal level in $G D P$ and an equity index. As a detailed derivation of the model is available elsewhere (Cohen, 2005), we shall avoid re-deriving it here and, instead, provide a brief summary of the notions that underlie it and the final outcome itself.

Beginning with the supposition that the value of a publicly traded security at a macro level [i.e. a broad equity index] depends on two parameters, one being the nominal $^{8}$ interest rate and the other time ${ }^{9}$, it can be shown that

$$
\ln S-b t=\Psi(b)
$$

where $S$ is the level of the index, $b$ is the interest rate, $t$ is an annual ${ }^{10}$ measure of time and $\Psi(b)$ is some function of $b$. There are several implications to the above relationship, more notably that $\Psi$ turns out to be a function of only $b$. This means that a plot of $\Psi$

\footnotetext{
${ }^{8}$ See Footnote 6.

${ }^{9}$ Refer to Cohen (2005) for a justification of how the two parameters were selected.

${ }^{10}$ The measure of time, $t$, is annual because the rate of interest is annual.
} 
against $b$ within some time range $t_{1}$ to $t_{2}$ should, in the absence of any outliers and structural shifts in the economy, yield a single, continuous curve, depending only on $b$.

Table 1 provides an example of how $\Psi$ is calculated from Equation 1, given $S$ and $b_{10}\left[b_{10}\right.$ is the 10-year US government bond rate] at any time, $t$. In the case of 1977 , for instance, where $t, b_{10}$ and $S$ are $2,7.21 \%$ and 104, respectively, $\Psi$ becomes

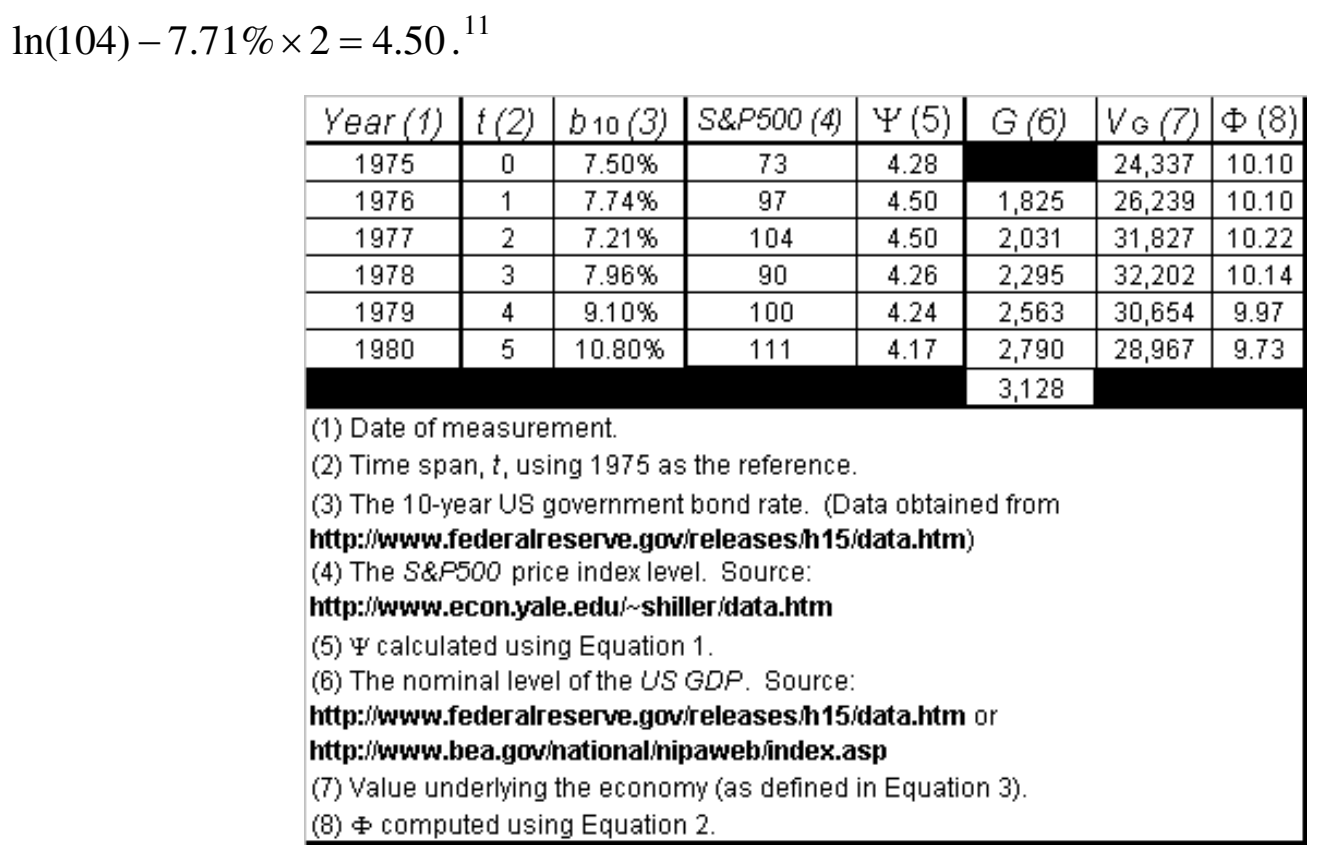

Table 1 - An example calculation of the function $\Psi$ in Column 5, given $t, b$ and $S$ in Columns 2-4, respectively. Columns 6-8, refer to the $G D P$, the treatment of which shall be discussed shortly.

The sample data in Table 1, starting with 1975, shows a time parameter, $t$, which begins at 0 , thus defining 1975 as the "reference" date. In fact, it does not matter what date is used as the reference, since the impact of this on the function $\Psi$ is linear and cancels out in subsequent calculations.

Table 1 contains three additional elements, all which are manifested in Columns 6-8 and pertain to the GDP. The parameter $G$ in Column 6 is the nominal level of the $U S$ $G D P$ in billions of dollars. The treatment of $G$, also described in detail in Cohen (2005), leads to the following relationship

$$
\ln V_{G}-b t=\Phi(b)
$$

\footnotetext{
${ }^{11}$ The reader may refer to Cohen $\mathbf{( 2 0 0 5 )}$ for more examples and charts.
} 
which is directly analogous to Equation 1 . Here, however, $V_{\mathrm{G}}$, which replaces the index, $S$, in Equation 1 represents the "value" of the economy at time $t$ and is defined as follows:

$$
V_{G}(t) \equiv \frac{G(t+1)}{b(t)}
$$

where $G(t+1)$ is the nominal GDP level one year ahead of $t$ and $b(t)$ is the interest rate at time $t$. Therefore, since $G$ is defined by units of earnings - $\$ / y e a r-V_{\mathrm{G}}$ will then acquire the units of value - \$ - thus allowing direct association between Equations 1 and 3, representing an equity index and the GDP's implied value, respectively

The reason for the 1-year gap in measurement time between $G$ and $b$ in Equation 3 has to do with how asset valuation is generally conducted in practice, which is based on forward looking earnings. In addition, if $t$ represents today, $t+1$ would be one year from today, thus deeming $G(t+1)$ a forecast and, hence, a speculation, just like an equity index [refer again to Cohen (2005) for a more detailed explanation].

An example of how $\Phi$ is calculated follows from the sample data in Table 1. In reference to 1977 , similar to the earlier illustrative calculation for $\Psi, V_{G}$ is computed as $G(t+1) / b(\mathrm{t})=2,295 / 7.21 \%=31,827$ leading, subsequently, to $\Phi(b)=\ln V_{G}-b t=\ln (10.22)-7.21 \% \times 2=10.22$.

\section{Impact of Different Maturities}

Although the interest rate, $b$, is a primary input to the model described above, its maturity has not been specified simply because it turns out to be irrelevant. What matters, though, is that $b$ must be represented by a government bond rate, since a government bond is typically free of any firm-specific risks [credit, liquidity, etc.].

Figures $2 \mathrm{a}$ and $2 \mathrm{~b}$ show $\Psi$ and $\Phi$, which relate to the $S \& P 500$ index and the $U S$ nominal GDP, respectively, plotted against $b$ for various $U S$ government bond maturities, namely 5, 10 and 30 years. The data range from 1975 to 2008 , inclusive. 


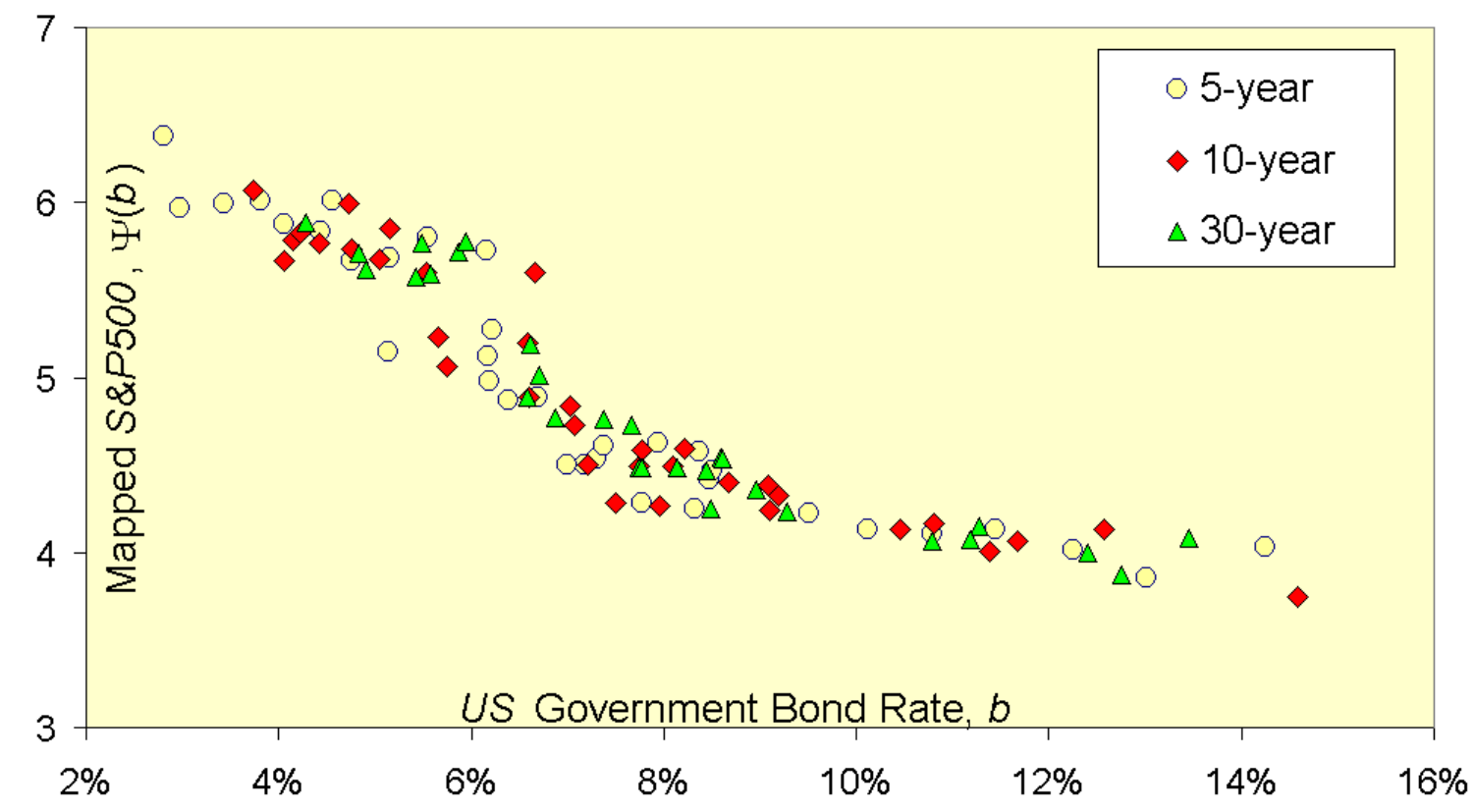

Figure $2 \mathrm{a}-$ The function $\Psi(b)$, defined by Equation 1 and based on the $S \& P 500$ index, is plotted here against $b$ for different maturities. It illustrates, firstly, the irrelevance of the maturity of $b$ and, secondly, the convergence of the data around $b$.

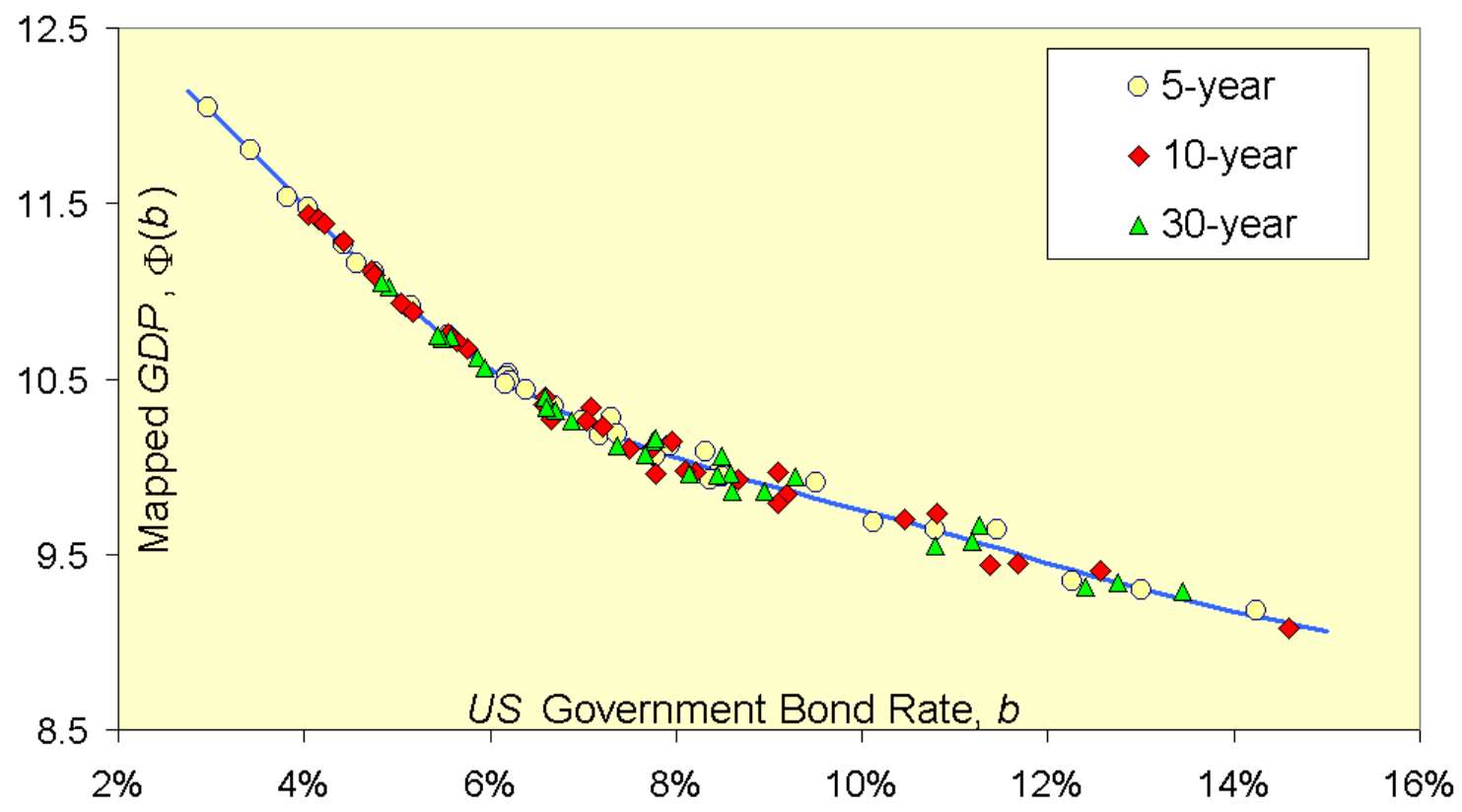

Figure $2 \mathrm{~b}$ - The function $\Phi(b)$, defined by Equation 3, is plotted here against $b$ for different maturities. Again, it depicts the irrelevance of the maturity of $b$, as well as the convergence of the data around $b$. The convergence is significantly tighter in this case than in Figure 2a, owing to the fact that the GDP is a realized number rather than speculated. The line is a best-fit polynomial through all the points. 
An important feature in Figures $2 \mathrm{a}$ and $2 \mathrm{~b}$ is the convergence of both functions $\Psi$ and $\Phi$ around $b$, which is implicit in how Equations 1 and 3 are derived. Never the less, $\Phi$ 's convergence is markedly tighter because the GDP is a realized number, whereas the equity index, which underlies $\Psi$ in Figure $2 \mathrm{a}$, is purely speculative. Another prominent attribute, particularly in Figure $2 b$, is that the impact of the different rate maturities is indeed irrelevant. $^{12}$

Finally, for estimation purposes, the mapped GDP data in Figure $2 \mathrm{~b}$ have been curve fitted with a polynomial of order 6 , characterizing $\Phi$ as a function of $b$ alone ${ }^{13}$. The single curve traverses all the different maturities considered, having taken into account their irrelevance.

\section{Defining the New GDP-based Benchmark}

With the above as background, we now proceed to construct the proposed GDP-based index for benchmarking purposes. For this, we refer to Figures $2 \mathrm{a}$ and $2 \mathrm{~b}$ and note the difference in the levels across $\Psi$ and $\Phi$, which is, exclusively, a result of the disparity in the measurement scales between the GDP and the equity index, here being the $S \& P 500$. Subtracting a constant, $\alpha_{\Phi \Psi}$, from the level of $\Phi$, thus shifting it down in parallel to coincide with $\Psi$, could easily circumvent this - i.e.:

$$
\Psi(b)=\Phi(b)-\alpha_{\Phi \Psi}
$$

which leads to

$$
\ln S=\ln V_{\mathrm{G}}-\alpha_{\Phi \Psi}
$$

\footnotetext{
${ }^{12}$ More examples of this nature, related to different jurisdictions, can be found in Cohen (2005).

${ }^{13}$ The $6^{\text {th }}$-order best-fit polynomial is given by $\Phi(b)=-9700930 \times b^{6}+5819200 \times b^{5}-1375820 \times b^{4}+$ $160458 \times b^{3}-9257.7 \times b^{2}+199.4 \times b+11.0$ with an $R^{2}$ of $99.4 \%$. The use of this choice of curve is driven primarily by the high $R^{2}$ it can achieve.
} 
upon combining Equations 1, 2 and 4. Minimizing the sum of squared errors between the data points in Figure $2 \mathrm{a}$ and the fitted curve in Figure $2 \mathrm{~b}$ gives $\alpha_{\Phi \Psi} \approx 5.41$.

Figure 3 depicts the fit between the curve in Figure $2 b$, as it is lowered by a constant value of 5.41 to the level of the data in Figure 2a. The comparison is satisfactory, attesting to a more direct relationship between the $S \& P 500$ index and $V_{G}$, as presented in Equation 5.

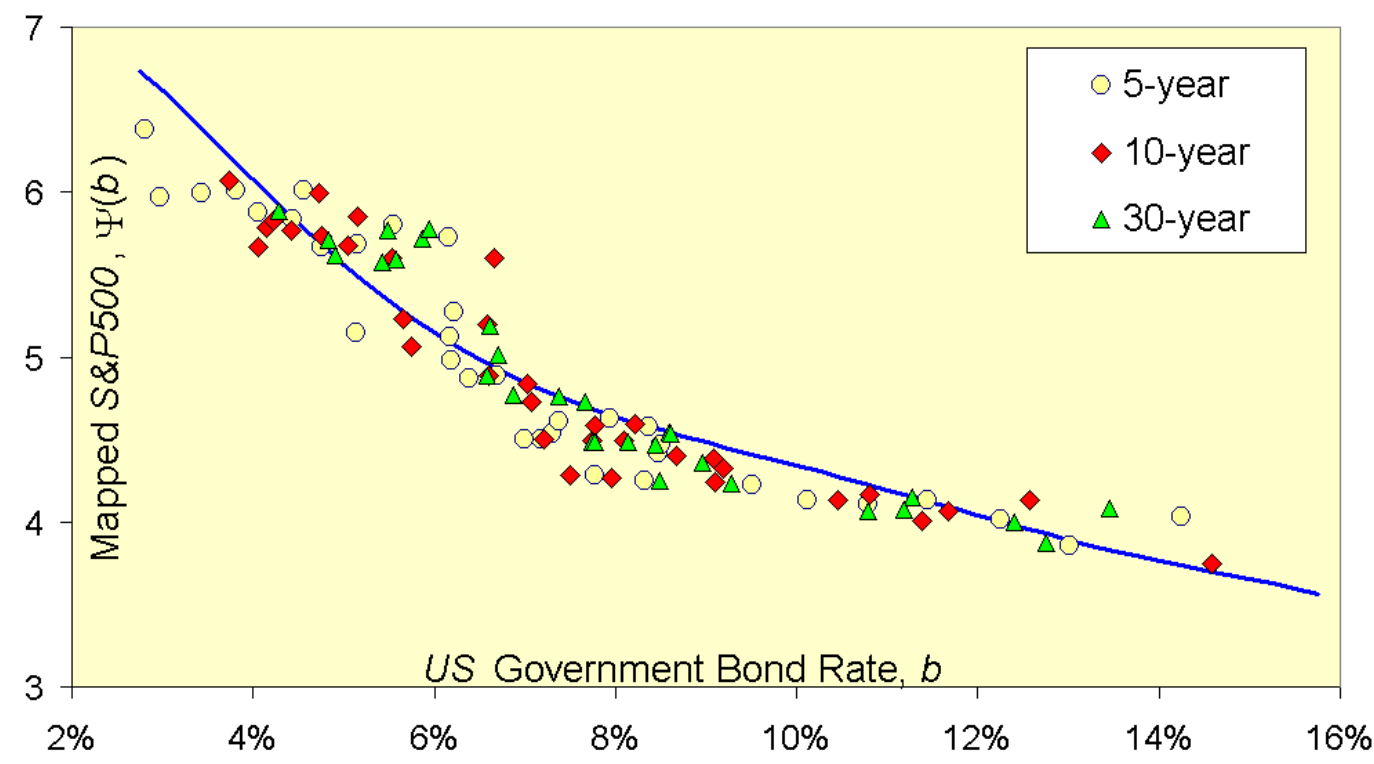

Figure 3 - Lowering the best-fit curve in Figure $2 b$ by a constant, $\alpha_{\Phi \Psi}$, equal to 5.41 and corresponding to the $S \& P 500$ index, and inserting into Figure 2a to compare with $\Psi(b)$.

The constant, $\alpha_{\Phi \Psi}$, will, of course, vary with other indices, depending on their scales relative to $V_{G}$. As an example, $\alpha_{\Phi \Psi}$ for the DJIA index turns out to be approximately 3.30, whose fit with the same line in Figure 2b, but parallel shifted, is portrayed in Figure 4. The strong similarity between Figures 3 and 4, relating to the $S \& P 500$ and DJIA indices, respectively, is due to the high correlation between the two indices. As high correlation among broad equity indices is generally the norm, the newly constructed GDP-based benchmark, corrected for $\alpha_{\Phi \Psi}$, could thus have large-scale applications. 


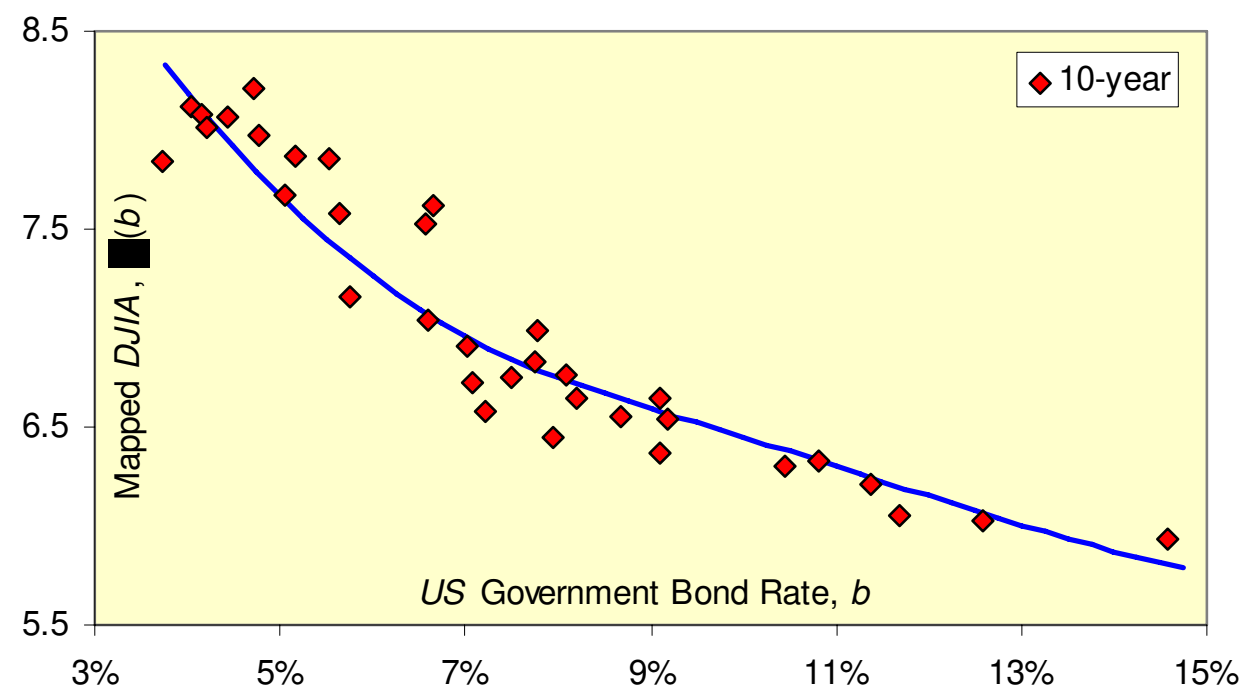

Figure 4 - Similar to Figure 3 and for the same time range, but for the DJIA index. The solid line is the best-fit line in Figure 2b, but with a parallel shift and an $\alpha_{\Phi \Psi}$ of about 3.30. To reduce the clutter, the figure includes the function $\Psi$ based on only $b_{10}$.

Next, upon exponentiating Equation 5, one can finally define $S$ as a $G D P$-based benchmark for any broad equity index. In the case of $S \& P 500$, for instance, incorporating 5.41 for $\alpha_{\Phi \Psi}$ leads to:

$$
S_{S \& P 500}=\exp \left(-\alpha_{\Phi \Psi}\right) \times V_{\mathrm{G}}=V_{\mathrm{G}} / 223.6
$$

where $S_{S \& P 500}$ represents the benchmark for the $S \& P 500$ index. Figures 5a-b compare the $S \& P 500$ index against its $G D P$-based benchmark, $S_{S \& P 500}$, both plotted against the year, ranging from 1975 to 2008. Being linear in scale, Figure 5a amplifies the differences at higher levels, whereas its logarithmic counterpart, Figure $5 \mathrm{~b}$, helps provide an objective comparison over the entire range of the time scale.

Prior to continuing on, we observe that the benchmark in Figures 5a-b is based on $b_{10}$ only, knowing well that $V_{G}$ in Equation 6 depends on the maturity that one ultimately chooses. The reason for focusing on a single rate, rather than all three considered here, is essentially to minimize the clutter in the graph and produce a clearer picture. 


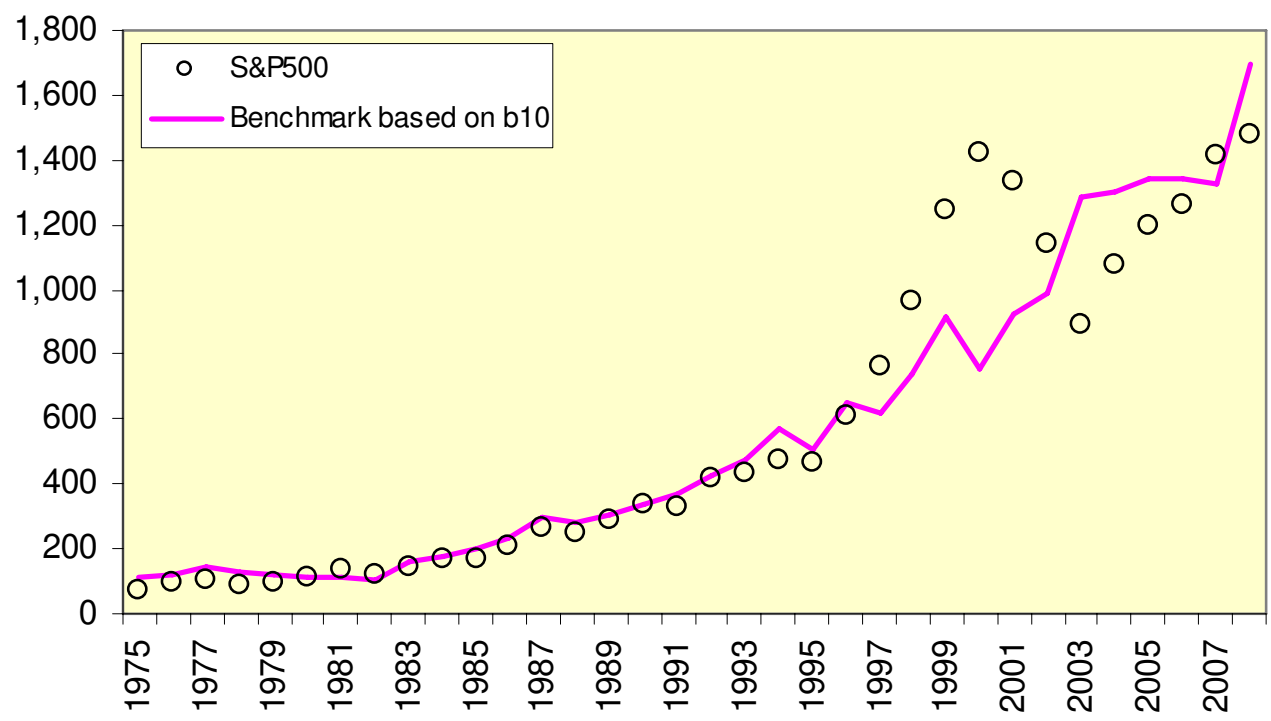

Figure 5a - Comparison of the new GDP benchmark, as calculated from Equation 6 and based on $b_{10}$, with the $S \& P 500$ index.

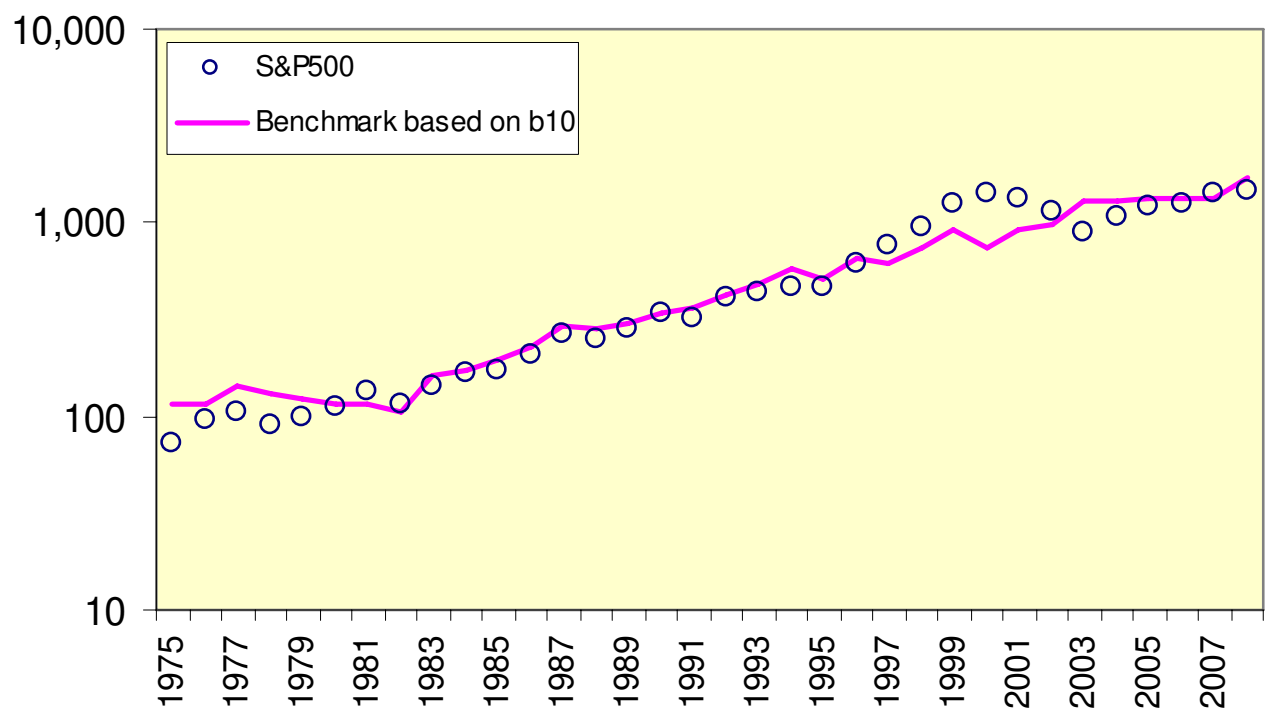

Figure $5 b$ - Same as Figure $4 a$, but in logarithmic scale.

Figure 6, on the other hand, is included here as well to depict how the proposed $G D P$ benchmark might vary depending on the different interest rate maturities that could go into calculating $V_{G}$. In this example, where the behavior of the benchmarks is displayed based on the 5 and 10-year maturities, we observe that, although the long-term trend is very similar in both cases, there appear to be gaps that separate them in certain time regimes. In this respect, it is worth mentioning that wider gaps in Figure 6 are 
caused by larger spreads in the corresponding yield curves, bearing in mind that this is perhaps an outcome of underlying economic uncertainties. In contrast, tighter gaps result from more horizontal yield curves, which, as discussed in Cohen (2006), could manifest periods of higher economic certainty. Therefore, as described here, the link between the maturity-induced gaps in the benchmarks and the spreads in the underlying yield curves could, potentially, explain how economic uncertainty is transmitted to equity markets.

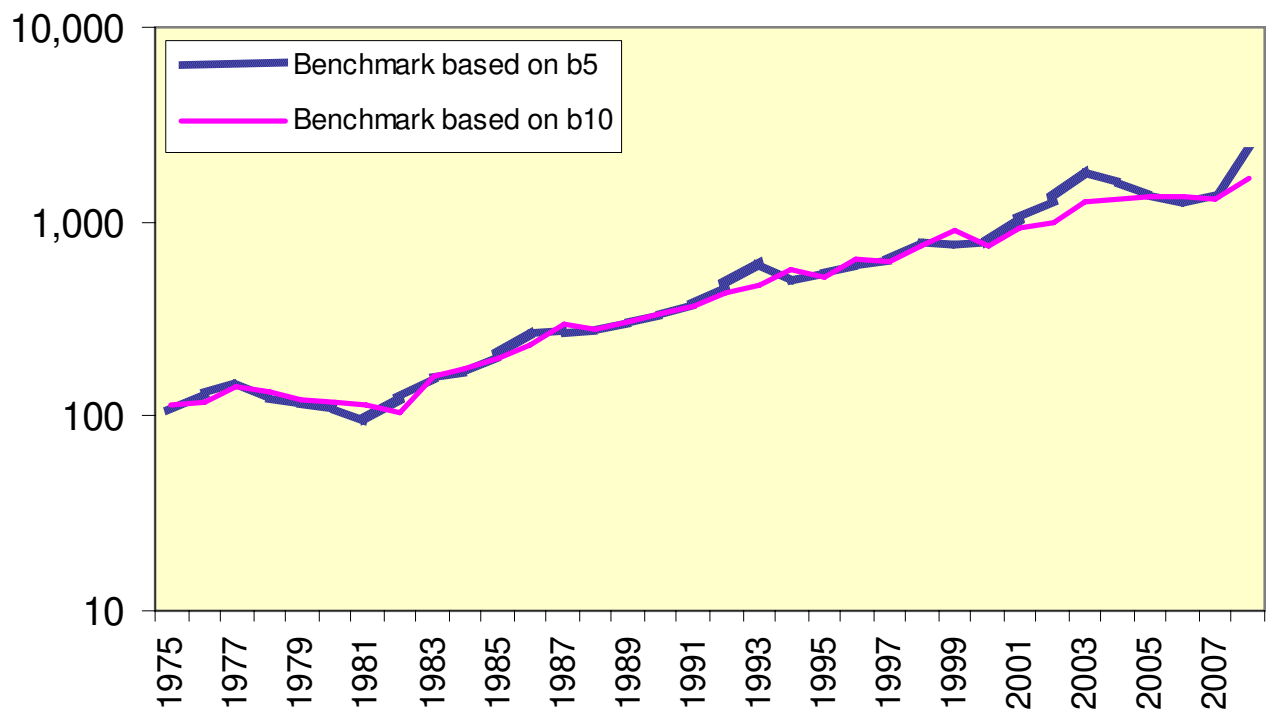

Figure 6 - Illustration of how the GDP-based benchmark, as given by Equation 6, differs depending on the maturity used to calculated $V_{G}$. The gaps between the benchmarks reflect the spread in the underlying yield curve, whereby larger spreads will lead to wider gaps and vice versa.

We finally refer back to Figures 5a-b and draw two important conclusions. Firstly, the issue of different measurement units, as discussed earlier and depicted by the use of two separate axes in Figure 1, is now resolved. The proposed method, effectively, places both the equity index and its benchmark into the same category of units, thereby allowing direct comparison between the two. Secondly, there is evidence of a better fit between the $S \& P 500$ index and its benchmark in Figures 5a-b than that in Figure 1. In essence, the index in Figures 5a-b appears to follow its GDP-based benchmark more closely than it does the GDP alone. The gaps in Figures 5a-b are, in addition, observed to occur in times well known for their characteristic market slumps [i.e. mid 1970's and between 2001-2004] and bubbles [1997-2000]. Clearly, therefore, the benchmark is also capturing the relative valuation effect that is embedded in its derivation. 
The appeal of the new benchmark goes beyond its satisfactory fit with the equity index it allegedly portrays, or its ability to highlight periods of over/under valuation of the index. Since the benchmark can be described in closed form, one should then also be able to estimate its duration - an important property - more easily and objectively, as it shall be demonstrated next.

\section{Calculating the Duration of the New Benchmark}

The duration of a financial instrument is normally defined as its sensitivity to the interest rate, all else constant. There are certain issues with this generic definition, never the less, which introduce a hurdle when it comes to practical implementation. These issues are two fold and provoked mainly by the following questions: (1) sensitivity to what interest rate in the yield curve and (2) how does one get around "all else constant", which is critical to the definition? Although these are non-issues when it comes to determining the duration of a bond, owing to the existence of a close-form valuation relationship, they are major when equities, equity indices and their related benchmarks are involved. This is likely due to the lack of an objective and closed-form equity valuation relationship, as well as the absence of some measure of maturity or investment horizon.

The literature, notwithstanding, does contain a number of works related to computing the duration of equity. Here, for instance, there is the notion that a combination of the book-to-market value and other ratios can provide a proxy for the [implied] duration [Dechow et al (2004) and Santa-Clara (2004)]. In the case of equity portfolios and, likewise, indices, the use of the Dividend Discount Model [DDM] for estimating duration appears to dominate, although different authors have offered tweaked versions of it. For example, while Casabona et al (1984) suggest directly implementing the $D D M$, Leibowitz et al (1989) propose incorporating a discount rate that takes into account an equity risk premium sensitive to both inflation and real rates. As related works are abundant in the literature, it is perhaps better, in the interest of space, not to delve deeply into them and, instead, proceed directly with obtaining the duration of the new GDP-based benchmark introduced here.

To get this, we combine Equations 2 and 5 and arrive at: 


$$
\ln S=\Phi(b)+b t-\alpha_{\Phi \Psi}
$$

where $S$ denotes the benchmark, as defined earlier, and $\alpha_{\Phi \Psi}$ a constant. Now, based on definition, we write the following

$$
D_{S} \equiv-\left(\frac{\partial \ln S}{\partial b}\right)_{t}
$$

with $D_{S}$ representing duration, $\partial \ln S / \partial t$ denoting sensitivity to the interest rate and the subscript $t$ symbolizing "all else constant." Substituting 7 into 8 finally yields:

$$
D_{S}=-\left[\frac{\mathrm{d} \Phi(b)}{\mathrm{d} b}+t\right]
$$

Given the above, along with an estimate for $\Phi(b)$ based on the curve fit in Figure $2 b$ [see polynomial in Footnote 13], one could, subsequently, compute the duration of the benchmark, $D_{S}$, without difficulty and in closed form. The choice of $b$ - be it $b_{5}, b_{10}, b_{30}$, or any other that is preferred - is also easily implementable, owing to the irrelevance property discussed earlier. How $D_{S}$ varies with the choice of $b$, therefore, would be indicative of the benchmark's sensitivity to the different maturities.

A sample calculation of the duration measure, as presented by Equation 9, is shown in Table 2. 


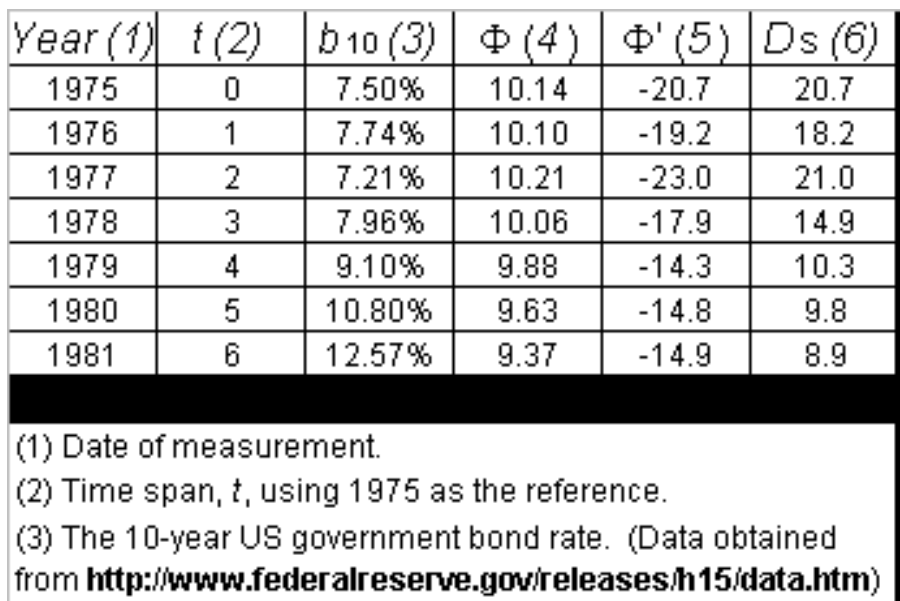

(4) Estimated $\Phi(b)$ based on the polynomial curve fit in Figure $2 \mathrm{~b}$. See Footnote 13.

(5) Derivative of $\Phi$ with respect to $b$, as calculated from the polynomial curve.

(6) Duration calculation based on Equation 9 .

Table 2 - Sample calculation of duration in accordance with Equation 9. 10-year rate has been selected here as the example.

The duration calculation in Table 2 has been extended to include the rates $b_{5}, b_{10}$ and $b_{30}$, as well as taken through to 2008 , with the outcome plotted in Figure 7 . Here, however, we have focused on 1990 onwards, so as to, once again, reduce clutter.

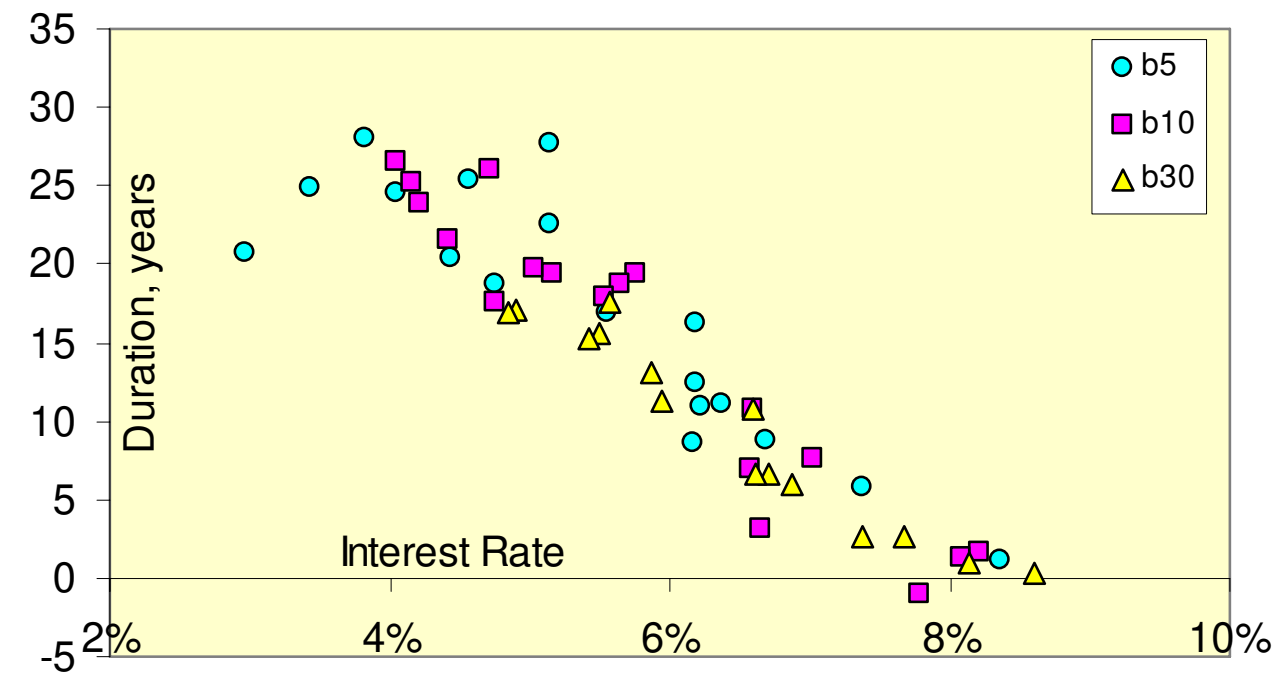

Figure 7 - Plot of the duration of the proposed benchmark, in accordance with the calculation procedure outlined in Table 7. Duration here is shown for different rates, namely $b_{5}, b_{10}$ and $b_{30}$, spanning from 1990 to 2008. 
Figure 7 contains some interesting features. Firstly, the irrelevance principle discussed earlier seems to apply to duration as well, as the durations computed for the different rate maturities considered here appear to, more or less, cluster uniformly around a common trend. This independence of maturity means, for instance, that the duration of the benchmark relative to $b_{5}$ would be equal to that relative to $b_{10}$, if the two rates were to be the same at a given time, $t$. This is not surprising because the fundamental input, $\Phi$, into calculating the benchmark's duration [see Equation 9] is independent of maturity. Secondly, there seems to be a clear trend that increases with decreasing interest rates, implying that the overall fall in interest rates, which has been observed since 1990, has led to a more rate-sensitive benchmark. Since the benchmark's long-term trend is a reflection of the major equity indices [i.e. see Figure 5b, for example, for the $S \& P 500$ ], one could conclude that indices have also, on the whole, experienced increased sensitivity to interest rates since 1990 .

To compare with other works, we display in Figure 8 the duration of the abovementioned benchmark plotted alongside that of the $S \& P 500$ index, the latter estimated using the method outlined in Blitzer et al (2008). The triangles, which summarize the outcome of this work, depict the duration values averaged over the three maturities considered herein, namely $b_{5}, b_{10}$ and $b_{30}$.

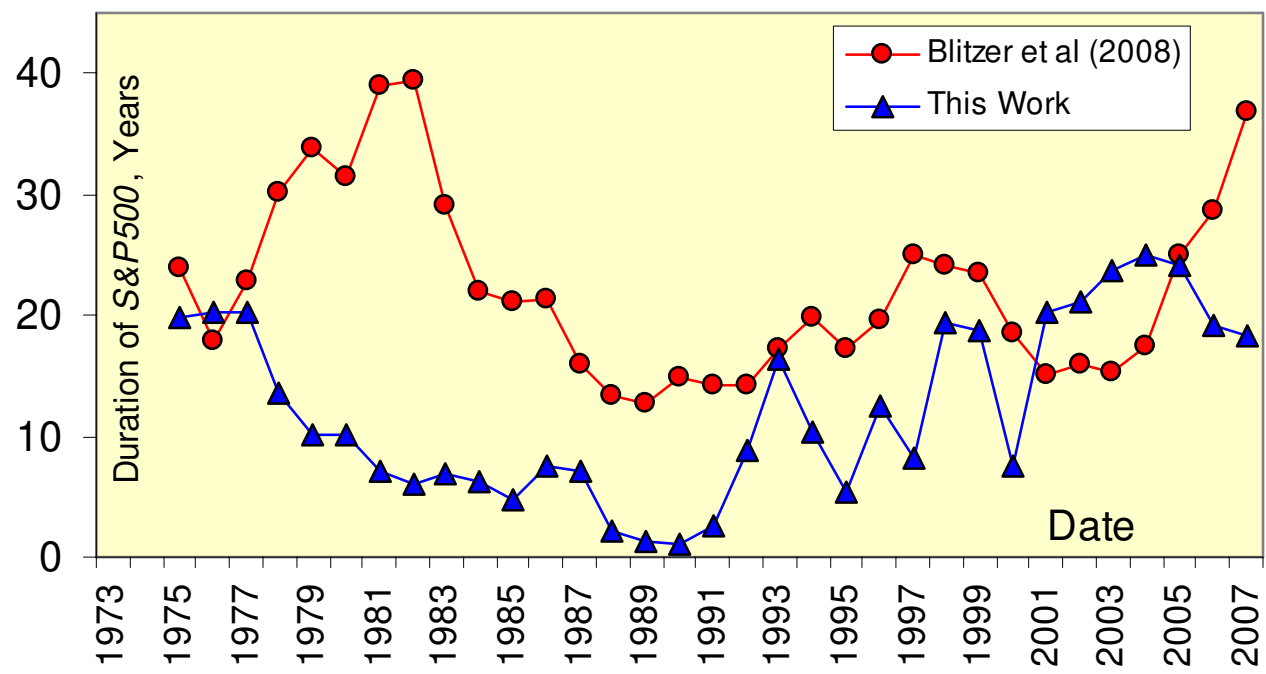

Figure 8 - Comparison of the duration of the proposed benchmark, as calculated from Equation 9, with that provided in Blitzer et al (2008). 
Prior to comparing the two, we should note that, firstly, the duration estimated in Blitzer et al (2008) relates to $S \& P 500$, which has been readily benchmarked here against the proposed index, with results shown in Figures 5a-b. Secondly, the method underlying the reference is based on the $D D M$, which implements certain assumptions on the risk premium, growth, corporate rating and maturity and, furthermore, incorporates moving averages and the like.

That said, the two lines in Figure 8 display certain disparities, as well as similarities. For instance, the estimate of Blitzer et al (2008) depicts a rising trend in duration during 1977-1981 and 2005-2007, whereas this work shows it to be falling. With the exception of these relatively short periods, the long-term trends appear to be, more or less, in line with each other. This goes along with the observation that none of the curves crosses zero at any point in time ${ }^{14}$. As for their estimated magnitudes, the duration measures do seem to correspond better with each other post 1992 rather than prior to. Altogether, our work shows the GDP-based benchmark to possess a higher volatility, perhaps due to the absence of any averaging scheme, moving and otherwise, within it.

We now outline another approach to estimating the duration of the newly proposed benchmark. Returning to Equation 7 and taking its total differential with respect to time, $t$, leads to:

$$
\frac{\mathrm{d} \ln S}{\mathrm{~d} t}-b=-D_{S} \frac{\mathrm{d} b}{\mathrm{~d} t}
$$

upon combining with Equation 9 and re-arranging. Equation 10 simply states that a plot of the left-hand side of Equation $10 v s$ the time rate of change in interest rate, $\mathrm{d} b / \mathrm{d} t$, all which can be assessed from available data [such as in Table 1], should provide information on the value of $D_{S}$. Figure 9 represents exactly this, where $\frac{\mathrm{d} \ln S}{\mathrm{~d} t}-b$ is plotted against $\mathrm{d} b / \mathrm{d} t$ over the time period 1975-2008 and for the three maturities

\footnotetext{
${ }^{14}$ One of the points in Figure 7 is clearly below the zero line, although it does not show up in the triangles in Figure 8. The reason for this is that the durations in Figure 8 are averaged over the three rate maturities,
} 
considered. ${ }^{15}$ Without going into any depth, it is noted that a best-fit straight line passing through all the points has a slope of -11.4 , suggesting that the average duration of the benchmark over the date range and maturities is about 11 years. This value is in good agreement with the overall average exhibited by the triangles in Figure 8. Equally important here is the absence of a statistically significant intercept, which is in full accord with the theoretical form of Equation 10.

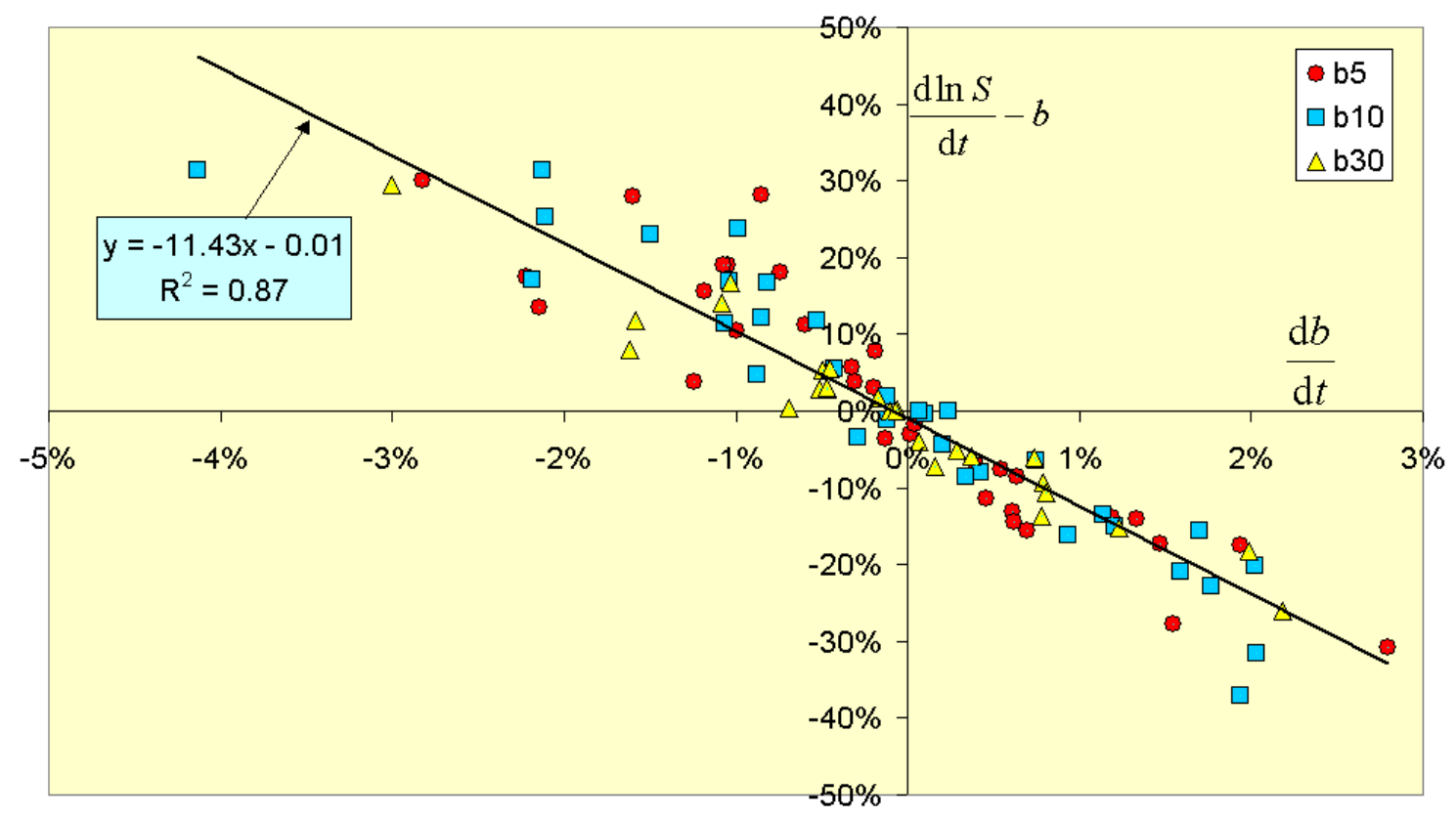

Figure 9 - Plot of $\mathrm{d} \ln S / \mathrm{d} t v s \mathrm{~d} b / \mathrm{d} t$ over the time period 1975-2008 and for the three maturities considered. According to Equation 10, the slope of the best-fit straight line should represent the average duration, here being about 11 years.

Finally, we include Figure 10, which is similar to Figure 9 but, for sake of clarity, focuses on a single maturity and a narrower time frame, namely $b_{10}$ and 1990-2008, respectively. The average duration over this time period is estimated at roughly 16 years, somewhat larger than the overall-average value indicated earlier. The reason for this is the rising trend in duration after 1990, as observed in Figure 8, and consistent, as well,

thus washing out this statistical anomaly. It should be pointed out that no similar anomalies where observed in relation to any of the other data points throughout the entire time frame 1975-2008.

${ }^{15}$ The time derivative, $\mathrm{d} / \mathrm{d} t$, is estimated here in annual difference, so that, in reference to Table $1, \mathrm{~d} b / \mathrm{d} t$ corresponding to 1976 , i.e. $t=1$, would be approximately the difference between $b(1976)$ and $b(1975)$, or $7.74 \%-7.50 \%=0.24 \%$. 
with the data in Figure 7, which portrays an increase in duration with falling interest rates.

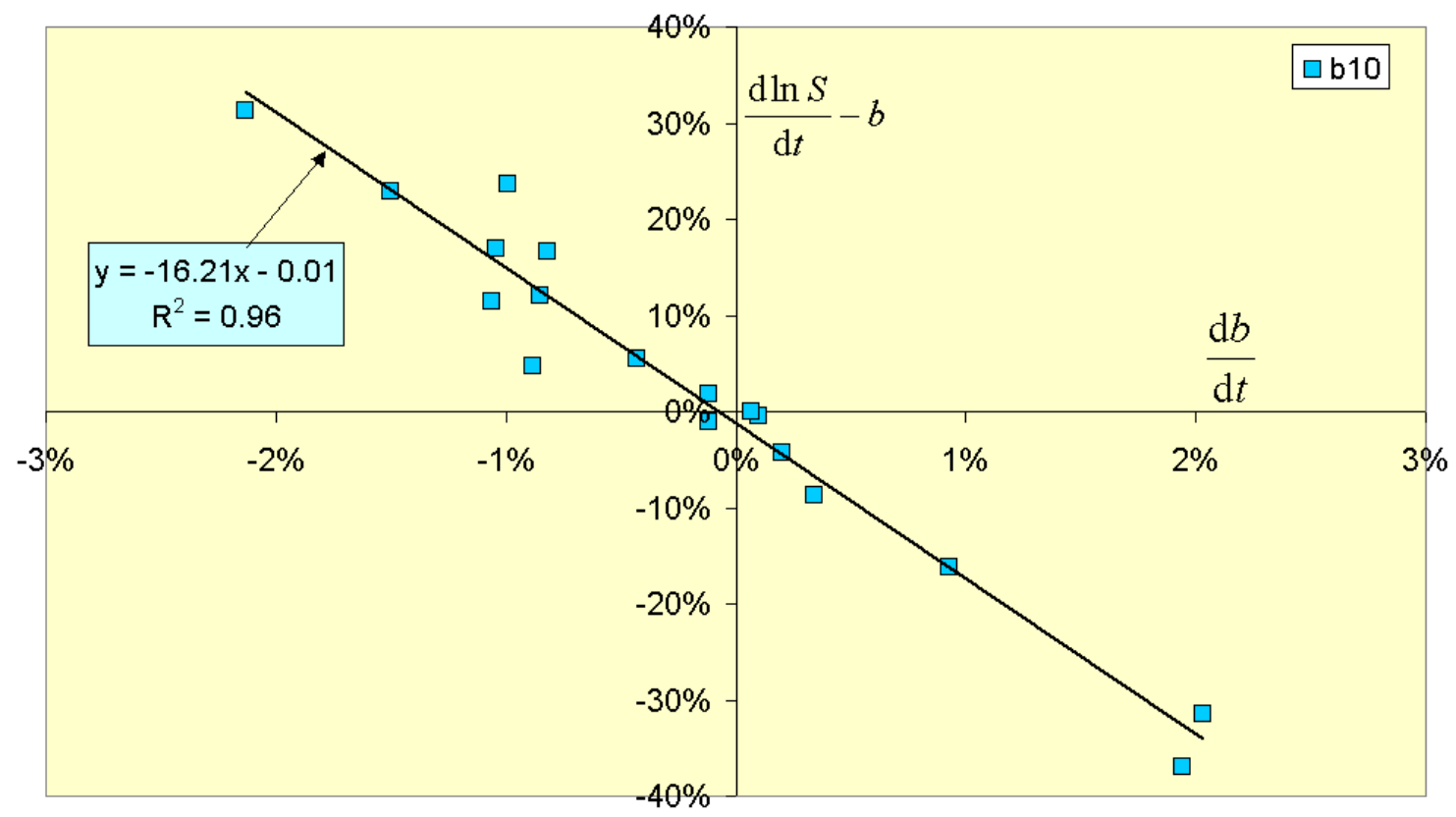

Figure 10 - Same as Figure 9, but focusing only on $b_{10}$ and the time frame 1990-2008. The average duration for $b_{10}$ throughout this time period is shown to be about 16 years. Also, the absence of a statistically significant intercept is consistent with the form of Equation 10.

\section{Conclusions}

This work addresses some of the issues surrounding the direct implementation of the GDP as a benchmark for broad equity indices and suggests a way for getting around them. It is shown here that representing the $G D P$ by its underlying value, rather than incorporating it on a standalone basis, produces not only a better fit as a benchmark, but also has other beneficial uses, such as providing a measure of relative valuation, whereby one could identify periods of under or over valuation of the index against which the benchmark is used.

Following on, the benchmark's duration is investigated as well, offering two distinctive and objective ways for measuring it, both of which seem to generate consistent results. While one approach establishes the trend, the other concentrates on estimating the average over a given time period, leading to the conclusion that the duration of the $U S G D P$-based benchmark relative to the $U S$ government rates averages 
at about 11 years over the time period 1975-2008, but increases to 16 years over the period covering 1990-2008. Moreover, the benchmark's duration appears to have risen since 1990 as interest rates have fallen gradually, a trend that seems to relate as well to the duration of the $S \& P 500$.

Finally, we re-iterate that this work touches only the surface of this very important area and, thus, leaves many questions unanswered. For instance, are the relationships and conclusions derived here universal and applicable equally across borders and over longer time horizons? Also, could certain situations, where a connection between the $G D P$-based benchmark and the underlying equity index is undoubtedly absent, point to underlying data issues or even the possibility of hidden market manipulations and inefficiencies? An extension of this work could potentially help address these questions and, perhaps, many others.

\section{References}

Blitzer, D.M., S. Dash and P. Murphy (2008) "Equity Duration - Updated Duration of the S\&P500", Standard \& Poor's Publication.

Casabona, P., F. J. Fabozzi, and J. C. Francis (1984) "How to Apply Duration to Equity Analysis", The Journal of Portfolio Management 10, pp.52-54.

Cohen, R.D. (2005) "The Relative Valuation of an Equity Price Index," Chapter 9 in The Best of Wilmott 2, P. Wilmott, Ed., John Wiley \& Sons, Ltd, pp. 99-132.

Cohen, R.D. (2006) “A Var-based Model for the Yield Curve," Wilmott Magazine, May issue, pp. 60-68.

Dechow, P.M, R.G. Sloan and M.T. Soliman (2004) "Implied Equity Duration: A New Measure of Equity Risk” Review of Accounting Studies 9, pp. 197-228.

Faugère, C. and J. Van Erlach (2006) "The Equity Premium: Consistent with GDP Growth and Portfolio Insurance", The Financial Review 41, pp. 547-564.

Honnerová, J. (2003) “Time Series Analysis of GDP and Market Indices”, Bulletin of the Czech Econometric Society 10, pp. 35-64.

Kerschner, E.M., T.M. Doerflinger and D.B. Murphy (1999) "What is the $S \& P$ " PaineWebber Investment Policy report.

Leibowitz, M.L., E.H. Sorensen, R.D. Arnott and H.N. Hanson (1989) “A Total Differential Approach to Equity Duration”, Financial Analysts Journal 45, pp. 30-37.

Santa-Clara, P. (2004) "Discussion of 'Implied Equity Duration: A New Measure of Equity Risk"”, Review of Accounting Studies 9, pp. 229-231. 